\title{
Experimental Demonstration of an All-Fiber Endless Polarization Controller Based on Faraday Rotation
}

\author{
J. Prat, J. Comellas, and G. Junyent
}

\begin{abstract}
An all-fiber device for the control of the state of polarization (SOP) is presented. The controller is high speed (its time response is less than $50 \mathrm{~ms}$ in the worst case), low loss (insertion losses are $2.5 \mathrm{~dB}$ independently of the input SOP), and low cost (is constructed with standard single-mode fiber). The polarization controller has been done using two Faraday rotators and works at $1550 \mathrm{~nm}$. We have also designed an algorithm that confers an endless performance to the device. The controller has been included in a $2.5 \mathrm{~Gb} / \mathrm{s}$ CPFSK optical coherent system and results are presented here.
\end{abstract}

\section{INTRODUCTION}

C OHERENT fiber-optic communication systems require the SOP of the received and local oscillator signals to be matched. This is often a major obstacle to achieve optimum performance. The problem is mainly due to the instability of the SOP when light propagates along standard single mode fiber. Although there is not a normalization of how fast a polarization controller should be, several works done [1]-[3] have dealt with the speed of polarization fuctuations in practical links, and we can conclude from them that fluctuations on installed fiber links are slower than a few $\mathrm{Hz}$, so $10 \mathrm{~Hz}$ can be taken as an upper limit.

Several solutions to this problem [4], [5] have been suggested in the past few years. Some of them are based on mechanically controlled birefringence effects (fiber squeezers and loops) and mechanical fatigue is its principal handicap. Integrated optic polarization controllers seem to be the best solution but they still present high insertion loss nowadays.

The polarization controller described in this work is based on the Faraday effect [6], [7]. It has its advantages in the fact that it is an all-fiber device and has no moving parts and only two variable elements, so it features low optical losses and fast response.

We begin with an explanation of the device's construction and its working principle in Section II. In Section III, the design of the driving algorithm is presented, with special attention given to the way that the device becomes endless. Finally, a description of the whole coherent system, and the results obtained after the integration of the controller in it are presented in Section IV.

Manuscript received August 3, 1995. This work was supported by the Spanish CICYT, project TIC92-1283-PB.

The authors are with the Signal Theory and Communications Department, Universitat Politècnica de Catalunya, UPC Campus Nord-D5, c/ Gran Capità Barcelona 08034, Spain.

IEEE Log Number 9415506.

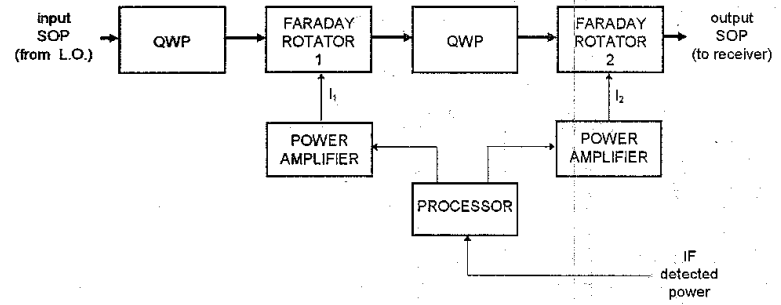

Fig. 1. Polarization controller block diagram.

\section{Construction of the Polarization CONTROLleR}

The controller that we have designed consists of two Faraday rotators (FR1 and FR2), with a fiber quarter wave plate (QWP) between them (as shown in Fig. 1). The rotators take advantage of the Faraday effect (circular birefringence is induced in fibers when a longitudinal magnetic field is applied) to change the incident SOP.

The first rotator tilts the linearly polarized incident light from the local oscillator laser to an arbitrary also linear SOP, by varying the rotator current $I$ [see Fig. 2(a)]. The QWP converts this tilt into ellipticity and finally the second rotator moves this elliptical SOP to reach the desired azimuth.

The Jones matrix of the Faraday controller is

$$
\begin{aligned}
M= & {\left[\begin{array}{lr}
\cos \theta_{2} & -\sin \theta_{2} \\
\sin \theta_{2} & \cos \theta_{2}
\end{array}\right] e^{-j(\pi / 4)}\left[\begin{array}{ll}
1 & 0 \\
0 & j
\end{array}\right] } \\
& \cdot\left[\begin{array}{rr}
\cos \theta_{1} & -\sin \theta_{1} \\
\sin \theta_{1} & \cos \theta_{1}
\end{array}\right]
\end{aligned}
$$

being $\theta_{1}$ and $\theta_{2}$ the angles rotated by FR1 and FR2, respectively. The detected Intermediate Frequency power depends on the SOP's angle difference $\eta$ as

$$
S=2 R^{2} P_{L O} P_{S} \cos ^{2} \eta
$$

being

$$
\begin{aligned}
\cos 2 \eta= & \cos 2\left(\alpha_{1} \alpha_{2}\right) \cos 2 \varphi_{1} \cos 2 \varphi_{2} \\
& +\operatorname{sen} 2 \varphi_{1} \operatorname{sen} 2 \varphi_{2}
\end{aligned}
$$

where $\alpha_{1}$ and $\alpha_{2}$ are the azimuths of the received and L.O. SOP's, respectively, and $\varphi_{1}$ and $\varphi_{2}$ are the corresponding ellipticities.

The rotated angle (when the incident light is linearly polarized) is given by

$$
\theta=V \cdot N_{f} \cdot N_{c} \cdot I
$$

where $V$ is the Verdet constant of the optical fiber. Because the effect is very low for $1550-\mathrm{nm}$ light, a great number of turns 

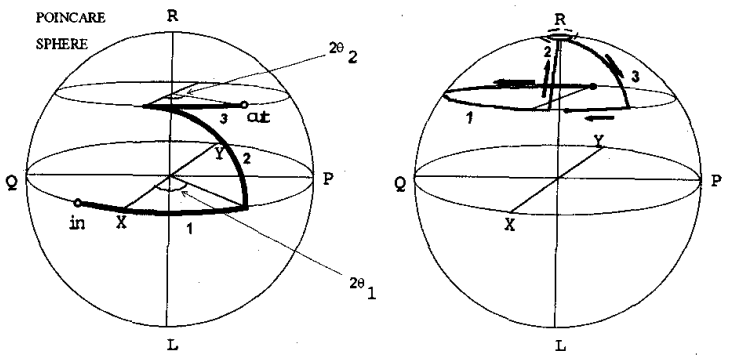

Fig. 2. Poincaré sphere representation of the (a) controller SOP transformation and (b) reset algorithm.

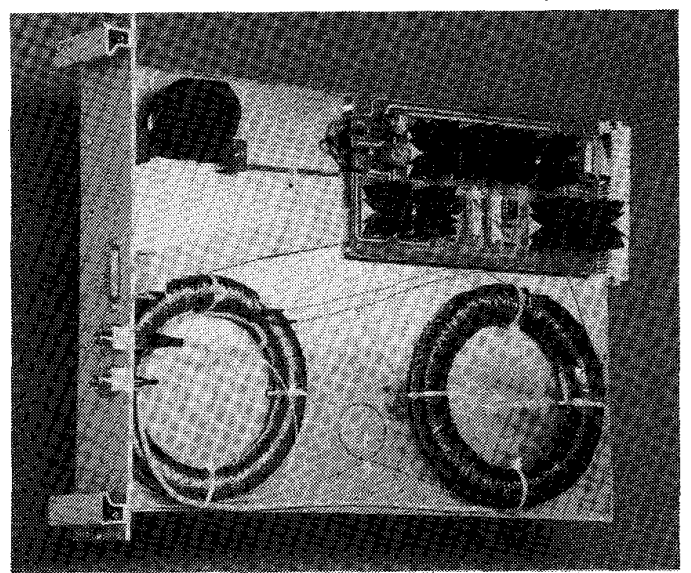

Fig. 3. Photograph of the Faraday polarization controller.

of fiber $\left(N_{f}\right)$ and copper wire $\left(N_{c}\right)$ is necessary to magnify the rotation. In our case, the first rotator has to reach $\pm 45^{\circ}$ and the second $\pm 90^{\circ}$. So, rotators consist of a single mode fiber wound in a 90 -mm-diameter reel with 600 and 1100 fiber turns, respectively. The controller insertion loss is $2.5 \mathrm{~dB}$ and is mainly due to nonideal winding process. To create the magnetic field there are 2000 and 2400 turns of copper wire with toroidal disposition. Power amplifiers supply the necessary currents (up to $0.9 \mathrm{~A}$ in both cases).

Some problems have to be taken into account during the construction process: the diameter of the reels can not be smaller because great optical losses appear, the fiber has to be twisted at a rate of approximately $120 \mathrm{rad} / \mathrm{m}$ to induce a degree of circular birefringence to suppress the effect of the intrinsic linear birefringence of the fiber, and microbendings during the winding process may dramatically increase the insertion losses. The electrical bandwidth of the rotators is about $700 \mathrm{~Hz}$. The photograph in Fig. 3 shows the constructed polarization controller.

\section{CONTROL Algorithm}

In a practical application, input SOP will vary continuously and randomly. So, a mechanism is necessary to maintain the two SOP's matched. The designed program varies both currents sequentially according to a simple maximum search algorithm. Problems may arise when one of the rotators reaches a limit, because the controller must be "endless" (the matching can not be lost at any instant). First rotator
TABLE I MulTichanNel 2.5-Gb/s CPFSK COHERENT SYSTEM

\begin{tabular}{ll}
\hline Bit rate & $2.48832 \mathrm{Gbit} / \mathrm{s}$ \\
Test signal & $\mathrm{NRZ} 2^{23}-1$ PRBS \\
Optical detection & Heterodyne, balanced \\
Error rate & $<10^{-9}$ \\
Optical fiber & Standard single-mode (G-652) \\
Wavelength & $1550 \mathrm{~nm}$ \\
Modulation format & $\mathrm{CPFSK}$ (index: 0.7$)$ \\
Demodulation & Differential \\
Intermediate Frequency & $4.3 \mathrm{GHz}$ \\
BW & $3.8 \mathrm{GHz}$ \\
Transmitted optical power & $0 \mathrm{dBm}$ \\
Receiver sensitivity & $-37 \mathrm{dBm}$ \\
Number of channels & 4 (minimum channel spacing: $13 \mathrm{GHz}$ ) \\
Ambient temperature range & $5^{\circ}-40^{\circ} \mathrm{C}$ \\
\hline
\end{tabular}

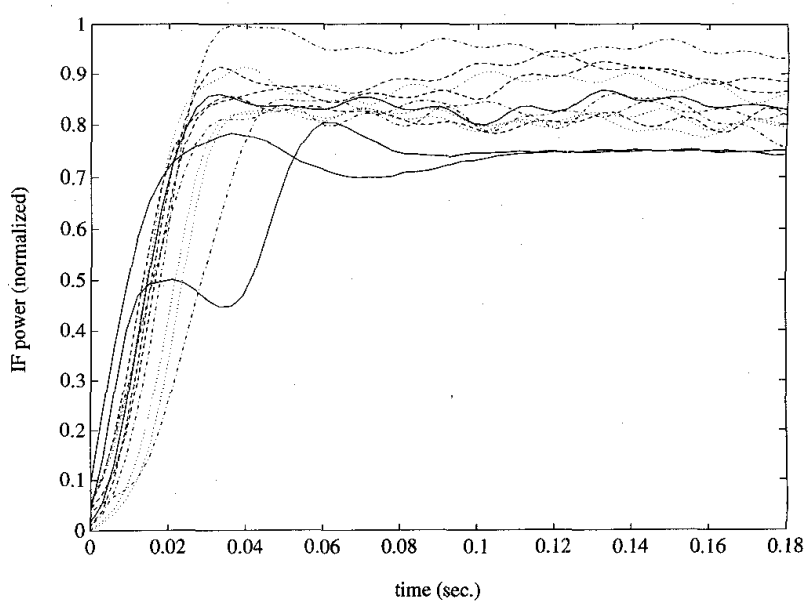

Fig. 4. System starting transient responses, in terms of the normalized IF power, from totally mismatched positions.

overcomes this problem as it will never pass its limit. To obtain endless tracking, when the FR2 current reaches a limit, FR1 is driven to its closest maximum [see Fig. 2(b)], so the polarization at the output of the QWP is circular. FR2 can then be reset without effect (it will be rotating a circular SOP) and the IF power will always keep over its $-3-\mathrm{dB}$ value. Hence, the continuous algorithm restarts.

\section{RESULTS}

The constructed controller has been included in a multichannel 2.5-Gb/s CPFSK coherent system [8], [9] whose main characteristics are detailed in Table I.

Measurements show that the polarization controller can track a variable input SOP continuously and IF power is maintained near maximum. Fig. 4 shows that the response time from a totally mismatched position is always less than $50 \mathrm{~ms}$, so the controller can follow drastic oscillations up to a rate of $10 \mathrm{~Hz}$, which, as we said above, is sufficient for practical links.

In Fig. 5 the IF power in the receiver when great circles in the Poincare sphere were forced on the input SOP by a liquidcrystal polarization variator is measured. When the controller is turned on the power is maintained near maximum (penalty $<0.5 \mathrm{~dB}$ ), even when resets occur (penalty $<3 \mathrm{~dB}$ ). This reset mismatch implies a global sensitivity penalty lower than $0,4 \mathrm{~dB}$, considering the typical SOP variation period and the 22-ms reset interval. 


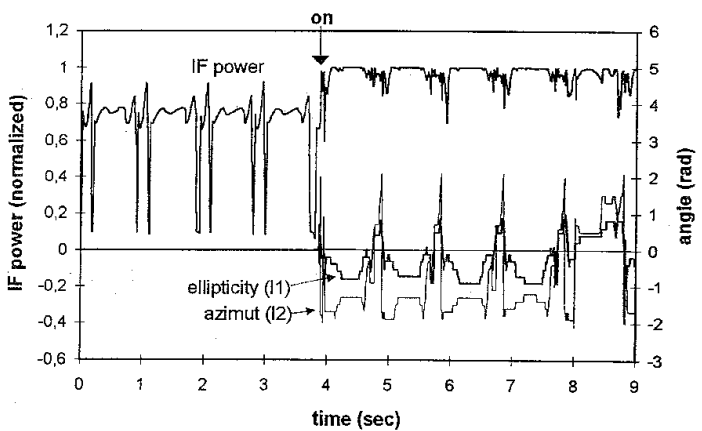

Fig. 5. Detected IF power against time over a 9-s interval (at $3.8 \mathrm{~s}$ the controller is switched on). The currents to the rotators are also plotted (expressed in terms of rotated angles), showing the behavior in reset instants.

\section{CONCLUSION}

Based on Faraday magnetooptic effect, the implemented polarization controller achieves endless tracking operation with fast response $(10 \mathrm{~Hz})$, and uses only two all-fiber variable elements. The obtained worst-case sensitivity penalty in the $1550-\mathrm{nm}$ coherent system is lower than $1 \mathrm{~dB}$. The measurements show that the controller automatically stabilizes the fluctuating output SOP of standard optical fiber with satisfactory performance.

\section{REFERENCES}

[1] Y. Namihira, Y. Horiuchi, S. Ryu, M. Kuwazuru, K. Mochizuki, and H. Wakabayashi, "Dynamic polarization fluctuation characteristics of optical fiber submarine cables under various environmental conditions," J. Lightwave Technol., vol. 6, no. 5, pp. 728-738, 1988.

[2] C. D. Poole, N. S. Bergano, H. J. Schulte, R. E. Wagner, V. P. Nathu, J M. Amon, and R. L. Rosenberg, "Polarisation fluctuations in a $147 \mathrm{~km}$ undersea lightwave cable during installation," Electron. Lett., vol. 23 , no. 21 , pp. 1113-1114, 1987.

[3] T. Pikaar, K. V. Bochove, A. V. Rooyen, H. Frankena, and F. Groen, "Nondeterministic endless control system for active polarization control," J. Lightwave Technol., vol. 7, no. 12, pp. 1982-1987, 1989.

[4] T. Okoshi, "Polarization-state control schemes for heterodyne or homodyne optical fiber communications," J. Lightwave Technol., vol. 3, no. 6, pp. $1232-1237,1985$.

[5] G. R. Walker and N. G. Walker; "Polarization control for coherent communications," J. Lightwave Technol, vol. 8, pp. 438-458, 1990.

[6] T. Okoshi, K. Kikuchi, and Y. Cheng, "A new polarization-control scheme for optical heterodyne receiver," in Proc. IOOC-ECOC' 85 1985, pp. 405-408.

[7] J. Prat, J. Comellas, and G. Junyent, "Endless Faraday polarization controller for a heterodyne optical system," in Proc CLEO'95, Baltimore, 1995, CTuL11.

[8] A. Aguilar, G. Junyent, J. Roldán, and J. Prat, "A 2.5 GBit/s CPFSK optical coherent system," in Proc. EFOC/LAN, London, 1991 pp. $402-408$.

[9] J. Prat, X. Portas, D. Brull, and G. Junyent, "Crosstalk statistics effect in dense spacing multichannel CPFSK coherent optical systems," in Proc. EFOC\&N'95, Brighton, UK, 1995, no. 139. 